\title{
Motion aftereffect of combined first-order and second-order motion
}

\author{
Maarten J van der Smagt $\uparrow$, Frans A J Verstraten, Eric B P Vaessen, Thomas van Londen, \\ Wim A van de Grind \\ Department of Comparative Physiology and Helmholtz Institute, Utrecht University, Padualaan 8, \\ NL-3584 CH Utrecht, The Netherlands; e-mail: maarten@salk.edu \\ Received 18 February 1999, in revised form 8 September 1999
}

\begin{abstract}
When, after prolonged viewing of a moving stimulus, a stationary (test) pattern is presented to an observer, this results in an illusory movement in the direction opposite to the adapting motion. Typically, this motion aftereffect (MAE) does not occur after adaptation to a second-order motion stimulus (ie an equiluminous stimulus where the movement is defined by a contrast or texture border, not by a luminance border). However, a MAE of second-order motion is perceived when, instead of a static test pattern, a dynamic test pattern is used. Here, we investigate whether a second-order motion stimulus does affect the MAE on a static test pattern (sMAE), when second-order motion is presented in combination with first-order motion during adaptation. The results show that this is indeed the case. Although the second-order motion stimulus is too weak to produce a convincing SMAE on its own, its influence on the SMAE is of equal strength to that of the first-order motion component, when they are adapted to simultaneously. The results suggest that the perceptual appearance of the sMAE originates from the site where first-order and second-order motion are integrated.
\end{abstract}

\section{Introduction}

Within the last decade, much research in the field of motion perception has focused on the distinction between first-order and second-order motion, as put forward by Cavanagh and Mather (1989). A problem for this dichotomy is that, although firstorder motion is quite clearly defined as spatiotemporal variations in luminance (or colour), second-order motion can be defined by spatiotemporal variations in a farrago of different stimulus attributes (such as contrast, spatial or temporal texture differences, stereo, etc).

The problem can be illustrated by studies of the motion aftereffect (MAE). The MAE is an illusory motion, which occurs when, after prolonged viewing of motion in a certain direction, a stationary scene is presented to the observer. The direction of the MAE is typically opposite to the direction of the adapting motion [see Anstis et al (1998) for a review]. Adaptation to second-order motion stimuli typically does not elicit a MAE when the test stimulus is a stationary scene (eg Derrington and Badcock 1985; see also Cavanagh and Mather 1989; Culham et al 1998). ${ }^{(1)}$ However, convincing MAEs on stationary test patterns ('static MAEs' or sMAEs) have been reported after adaptation to stereo-defined (cyclopean) motion (eg Patterson et al 1994; Bowd et al 1996). Moreover, in these studies cross-adaptation occurred between luminance-defined (firstorder) and stereo-defined stimuli, which led the authors to suggest that a common neural substrate may well be responsible for the processing of both first-order and stereo-defined stimuli.

- Present (and correspondence) address: Vision Center Laboratory, The Salk Institute for Biological Studies, 10010 North Torrey Pines Road, La Jolla, CA 92037-1099, USA.

(1) One exception can be found in a study by Mather (1991), where moving, contrast-reversing bars did elicit a MAE, though much weaker than moving first-order bars. However, the perceived MAE might also conceivably be attributed to residual first-order motion information in the adaptatation stimuli. 
In recent years, a new class of test stimuli has come to be widely used: the directionally ambiguous 'flicker' or 'dynamic' test stimuli (eg von Grünau and Dube 1992; Hiris and Blake 1992; Ledgeway 1994). With these dynamic test stimuli, MAEs from adaptation to second-order motion have been demonstrated convincingly (eg McCarthy 1993; Ledgeway 1994; Nishida and Sato 1995; but see Cropper and Hammett 1997), adding to evidence from motion-threshold elevation studies (eg von Grünau 1986; Turano 1991; Holliday and Anderson 1994). Strong cross-adaptation between first-order and second-order stimuli has been shown as well (eg Turano 1991; Ledgeway and Smith 1994).

To account for these findings, Nishida and Sato (1995) proposed a mechanism based on an earlier model of motion perception and integration by Wilson et al (1992). The model (consisting of a sensory layer, an opponent energy layer with both firstorder and second-order units, and an integration layer) allows for multiple sites of adaptation along the motion pathway (Mather and Harris 1998). Nishida and Sato (1995) postulated that the sMAE originates at the low-level, first-order motion detection system (ie sensor layer or first-order units of the opponent energy layer). ${ }^{(2)}$ Since a 'dynamic MAE' (dMAE) occurs after adaptation to second-order as well as first-order motion, the dMAE was therefore assumed to reflect adaptation at a higher level, where first-order and second-order motion are integrated (ie integration layer). In accordance with this idea, interocular transfer (IOT) of the dMAE was shown to be near $100 \%$, whereas an sMAE transfers only partially (Raymond 1993; Nishida et al 1994).

There are, however, some problems with the views outlined above. First of all, complete IOT of the dMAE seems to depend upon the parameters used, and is not too general a result (eg Steiner et al 1994; see also the Appendix to this paper). Moreover, Verstraten et al (1998) showed that dynamic visual noise when used as a test pattern, elicits strong MAEs after adaptation to high-speed motion, whereas for slow adaptation MAEs are less strong compared to those obtained in tests with static visual noise. Adaptation in both cases contains predominantly first-order motion, so even in the low-level first-order motion detection system there seems to be a distinction between sensors of which the adaptation is revealed either by dynamic or by static test patterns. Van der Smagt et al (1999) showed that, after adaptation to transparent motion consisting of a fast and a slow component, testing with a combination of static and dynamic visual noise resulted in a transparent MAE, with the dynamic and static visual noise components directed opposite to the fast and slow adapting components respectively. At least in this case, the dynamic MAE does not seem to originate from an integration layer.

Furthermore, Wilson et al (1992) showed that the perceived direction of a moving 'type-II plaid' (the result of two superimposed sinusoidal gratings which are oriented such that the resulting plaid direction lies outside the two component directions), is well described by a vector sum of the first-order and the second-order motion components in the stimulus. This is of interest, since the sMAE of this kind of plaid appears to be directed opposite to its perceived direction (Burke and Wenderoth 1993; Alais et al 1994). Consequently, the sMAE can also reflect either adaptation of second-order motion sensors, or adaptation of units at a higher-level integration stage.

In summary, a dMAE occurs after adaptation to either (fast) first-order motion, second-order motion, or a combination of the two. An sMAE seems to occur only for (slow) first-order motion, but not for second-order (except stereo-defined) motion. However, adaptation to a combination of first-order and second-order motion might result in an SMAE in which the adaptation to both the first-order and second-order motion components is visible (see above). We will show here that this is indeed the case, although the second-order motion stimuli are ineffective in eliciting a MAE when

(2) Note that the resulting output of adaptation in the sensor or opponent energy layer has to be processed by the integrator layer, because the sMAE of bivectorial motion is unidirectional and opposite to some integration of the adapting vectors (Mather 1980; Verstraten et al 1994). 
presented in isolation. The results indicate that static MAEs might also reflect adaptation at higher levels in the visual motion pathway. We will discuss the implications of this finding for the distinction between dynamic versus static MAEs.

\section{Experiment 1}

\subsection{Rationale}

This experiment is based on an experiment by Alais et al (1995). In that experiment observers had to indicate the direction of a moving textured grating (see figure 1a) and of the MAE after adaptation to such a grating (tested with either a stationary textured grating, or stationary random texture across the entire aperture). These textured gratings were originally devised by van den Berg and van de Grind (1993) in order to decouple motion direction and grating orientation. The grating can move in oblique directions while the orientation of the grating is kept constant (horizontal). Thus, motion direction and grating orientation are two independent variables. Alais et al showed that, although the motion direction judgments for these gratings are near veridical (see also van den Berg and van de Grind 1993), the (static) MAEs are judged closer to perpendicular by as much as $25^{\circ}$. In these stimuli, the direction of motion of the (horizontal) grating is actually defined by the motion of the texture (which is 'glued' to the bright regions of the square-wave grating: see figure 1a). However, since only the bright regions are textured, there is a luminance border present as well, which moves perpendicular to the grating orientation. Alais et al thus concluded that these textured gratings are in fact bivectorial stimuli, activating two populations of direction-selective units (that differ in spatial tuning) during adaptation, which is reflected in the MAE direction.

In the present experiment, we extend the experiment by Alais et al into the domain of second-order motion. Essentially, we add two conditions in which the luminance difference between the original textured ('bright') region and the nontextured ('dark') regions is removed. Any consistent motion information perpendicular to the grating

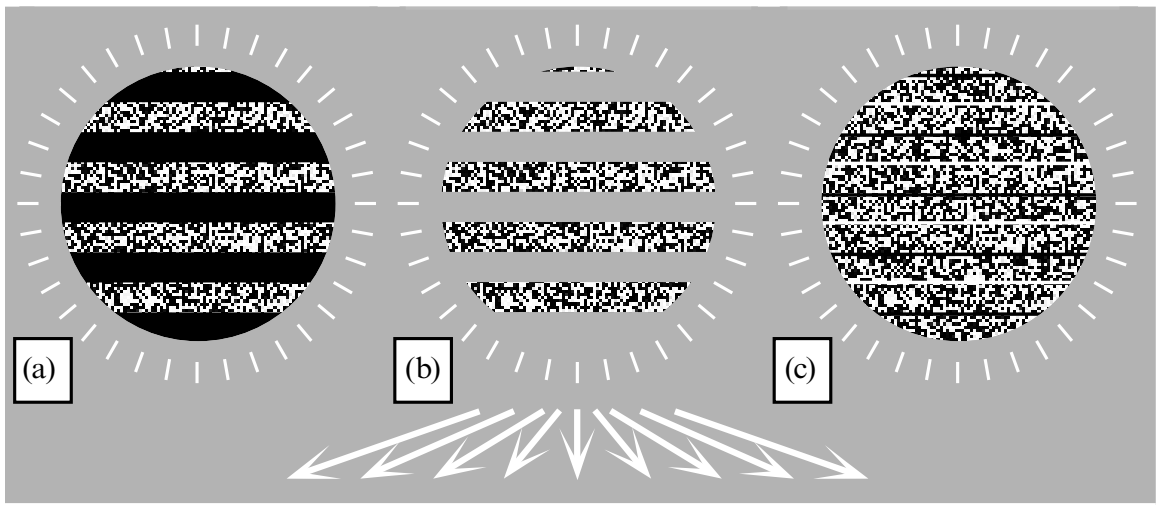

Figure 1. Schematic representation of the stimuli used in experiment 1. White arrows indicate the possible motion directions of the horizontally oriented gratings. The texture on the gratings ensures that the direction of motion is perceived near veridical. The white lines are $10^{\circ}$ tick marks. (a) The Luminance condition, which was similar to the stimuli used by Alais et al (1995). When the grating moves for instance down-left, the texture moves down-left and the grating is perceived to move down-left. However, a luminance border also moves straight down, which influences the perceived MAE direction. (b) The Contrast condition is similar to the Luminance condition, except for the dark background, which is now grey with the same luminance as the mean luminance of the textured region. Only a contrast border moves straight down during adaptation. (c) The Texture condition, where the background consists of stationary texture. The dark and bright horizontal lines are for illustration purposes only, to distinguish the moving regions from the background. During adaptation, the only downward-moving component is a border between dynamic and static texture. The test pattern is one large field of stationary texture. 
orientation is now defined by motion of texture boundaries only. If this second-order motion does not contribute to the sMAE, the perceived direction of the SMAE will be directly opposite to the perceived motion direction during adaptation. A deviation away from the direction resulting from first-order motion alone is expected only if the second-order motion component does influence the sMAE.

\subsection{Methods and procedure}

The stimuli were generated on custom image-generation hardware, controlled by a Macintosh computer and presented on a CRT display (ElectroHome EVM-1200, P4 phosphor, base display rate $90 \mathrm{~Hz}$ ). The display screen was $14 \mathrm{~cm} \times 14 \mathrm{~cm}$ square, one pixel subtending $0.55 \mathrm{~mm}$. Viewing distance was $2 \mathrm{~m}$, resulting in a pixel size of $0.94 \mathrm{~min}$ of arc. The stimuli were viewed through a circular aperture of 136 pixels $(2.15 \mathrm{deg})$ in diameter. Tick marks, indicating $10^{\circ}$ intervals, were placed outside the perimeter of the aperture to increase the accuracy of the direction judgments. Outside the aperture, the stimulus window had a luminance of $50 \mathrm{~cd} \mathrm{~m}^{-2}$ (grey) except for the pointer and the tick marks which were brighter $\left(95 \mathrm{~cd} \mathrm{~m}^{-2}\right)$. A fixation marker, which consisted of two centred dots (one bright, 4 min of arc in diameter, occluding part of a grey dot, $11 \mathrm{~min}$ of arc in diameter) was presented in the centre of the aperture.

2.2.1 Stimuli. There were three stimulus conditions which are depicted in figure 1 (see below for a detailed description): (i) A Luminance modulation, which replicates the experiment by Alais et al (1995), containing bivectorial first-order motion; (ii) a Contrast modulation, where the motion component perpendicular to the grating orientation is only defined by a difference in texture contrast; (iii) a Texture modulation, where the perpendicular motion component is defined only by the different (temporal) characteristics of the regions of the grating.

(i) The Luminance condition (figure 1a) consisted of a horizontal square-wave grating ( $50 \%$ duty cycle; 2.5 cycles $\mathrm{deg}^{-1}$ ) of which the bright region consisted of random pixels which had a $50 \%$ chance of being dark $\left(5 \mathrm{~cd} \mathrm{~m}^{-2}\right)$ or bright $\left(95 \mathrm{~cd} \mathrm{~m}^{-2}\right)$. The mean luminance of this region thus was $50 \mathrm{~cd} \mathrm{~m}^{-2}$. The pixels moved along with the grating, as if 'glued' to the bright regions of the grating. The dark region of the grating had the same luminance as the dark pixels. Since in this condition there is a luminance border that moves in a direction perpendicular to the grating orientation during adaptation, one would expect the MAE to deviate from being directly opposite to the perceived motion direction, as was the case in the Alais et al (1995) experiment. The MAE is expected to arise from a weighted vector summation of this perpendicular component and the moving texture. All observers performed in this condition.

(ii) The Contrast condition (figure 1b) was identical to the Luminance condition except for the luminance of the 'dark' region. Here, this luminance was identical to the mean luminance of the textured region. Therefore there is one first-order motion component (the oblique motion of the texture) and one second-order motion component (perpendicular to the grating orientation). One would expect the MAE of this stimulus to be exactly opposite to the perceived adaptation direction, since in the first-order domain the adaptation stimulus has become unidirectional. Any consistent deviation in MAE direction from opposite to the perceived motion can only occur when the second-order motion component (perpendicular to the grating orientation) contributes to the MAE as well. Four of the observers performed in this condition.

(iii) The Texture condition (figure 1c) differed from the other conditions. This stimulus is easier explained if one interprets the texture regions of the other stimulus conditions as textured bars moving over a background that is either dark or grey (mean luminance). In the Texture condition then, these textured bars move over a background consisting of stationary texture with the same pixel characteristics as the texture of the moving bars. For this condition, there is again no first-order motion component perpendicular to the 
orientation of the bars. However, there is a moving border between stationary and dynamic texture. Thus, as in the Contrast condition, there is a first-order (oblique) motion component as well as a second-order (perpendicular) motion component in the stimulus. Again, only if the second-order motion component contributes to the MAE, one would expect the MAE to deviate from being exactly opposite to the perceived direction. Three of the observers performed in this condition.

The movement of the horizontal gratings was created by displacing the grating 1 pixel per 2 frames downwards (perpendicular to the grating orientation), and 0 to 4 pixels either to the left or to the right (parallel to the grating orientation), the direction and size of the displacements being randomised. The resulting directions and speeds are summarised in table 1 .

Table 1. The combinations of perpendicular and parallel displacement used in experiment 1 and the resulting direction and speed of the adaptation stimulus. The perpendicular displacement was always 1 pixel. Perceived motion and MAE directions for positive and negative displacements (right and left of straight-down) were mirrored and pooled in the analysis of the data.

\begin{tabular}{lccccccccc}
\hline \multicolumn{10}{c}{ Parallel displacement/pixels } \\
\cline { 2 - 9 } & -4 & -3 & -2 & -1 & 0 & 1 & 2 & 3 & 4 \\
\hline Direction $^{\circ}{ }^{\circ}$ & -76 & -72 & -63 & -45 & 0 & 45 & 63 & 72 & 76 \\
Speed/deg s $^{-1}$ & 2.94 & 2.25 & 1.59 & 1.01 & 0.71 & 1.01 & 1.59 & 2.25 & 2.94 \\
\hline
\end{tabular}

2.2.2 Procedure. Two of the authors and four naïve observers participated in the experiment, which was performed in a room with dimmed light conditions. The observers were asked to indicate the perceived direction of the adapting motion as well as that of the MAE. They used the computer keyboard to place a pointer outside the perimeter of the aperture, in such a fashion that a line from the centre of the screen (fixation point) through this pointer would be parallel to the perceived direction. The pointer could be moved clockwise or anticlockwise in $1^{\circ}$ or $10^{\circ}$ steps. While fixating on the fixation dot, observers indicated the direction of motion, after which they adapted to the moving stimulus for $30 \mathrm{~s}$ more. Then the motion stopped and the observers indicated the direction of the MAE. A $60 \mathrm{~s}$ pause was given between trials, during which period the observers were free to move their eyes and look around. Since pilot experiments showed most MAEs for the Texture condition to be very weak (possibly caused by the increased amount of correspondence noise in the adaptation stimulus), for this stimulus the observers adapted for $60 \mathrm{~s}$ while the pause between trials was reduced to $30 \mathrm{~s}$. For all stimulus conditions, each direction was tested 10 times. For each parallel displacement size, the perceived motion and MAE directions left of straight down were mirrored across the perpendicular and averaged together with those right of straight down.

\subsection{Results}

As an example, the results of the Luminance condition for observer EV are shown in figure 2. Note that we added $180^{\circ}$ to the MAE directions in order to be able to compare them directly to the perceived motion direction. Consequently, when perceived motion and MAE are equal in the graph, they were perceived exactly opposite to each other in the experiments. Figure 2 shows that, while the perceived direction of the moving grating is near veridical for all parallel displacements, the perceived MAE direction does not follow the same pattern. For parallel displacements of 0 and 1 pixels every 2 frames (which results in stimulus directions of $0^{\circ}$ or perpendicular to the grating orientation, and $45^{\circ}$ ), the MAE direction is about opposite to the perceived motion direction. For larger parallel displacements (2, 3, and 4 pixels, resulting in $63^{\circ}, 72^{\circ}$, 


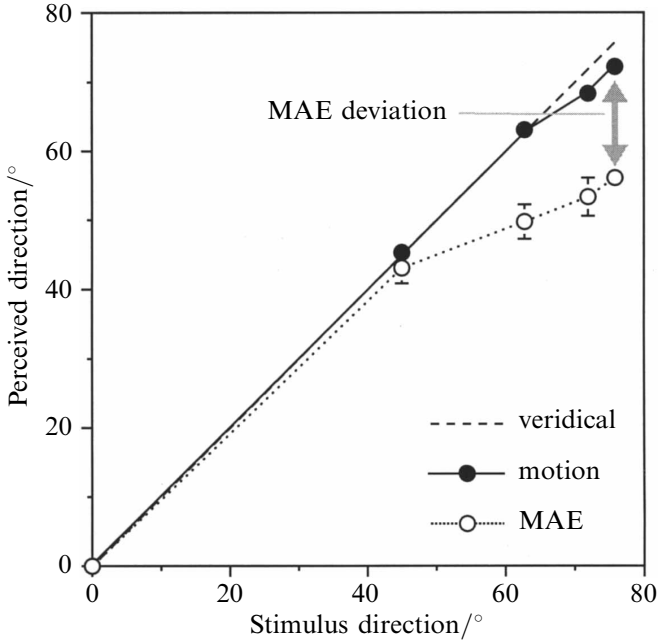

Figure 2. Example of the judgments by one observer (EV) of perceived motion direction and MAE direction in the Luminance condition. Perceived direction is plotted as a function of the stimulus direction. The dashed line represents the veridical direction, filled circles the perceived motion direction, and open circles the perceived MAE direction. Error bars depict the SEM. Although motion direction was perceived near veridical, the perceived MAE direction differed considerably. The difference between perceived motion and MAE direction (MAE deviation) is plotted for all conditions in figures 3 and 4.

and $76^{\circ}$ ), the MAE direction deviates from the perceived motion direction toward the perpendicular by as much as $16.3^{\circ}$ for the $76^{\circ}$ condition. A $t$-test shows the difference between motion and MAE directions to be significant $(t=5.11, p<0.01$ for the $63^{\circ}$ condition; $t=5.37, p<0.001$ for the $72^{\circ}$ condition; $t=13.27, p<0.001$ for the $76^{\circ}$ condition). Since this MAE deviation is the main point of interest here, we plot this deviation in figures 3 and 4 for the different conditions.

In figure 3, the mean deviation of the perceived MAE from the perceived adaptation direction is shown for each observer for the Luminance and Contrast conditions.

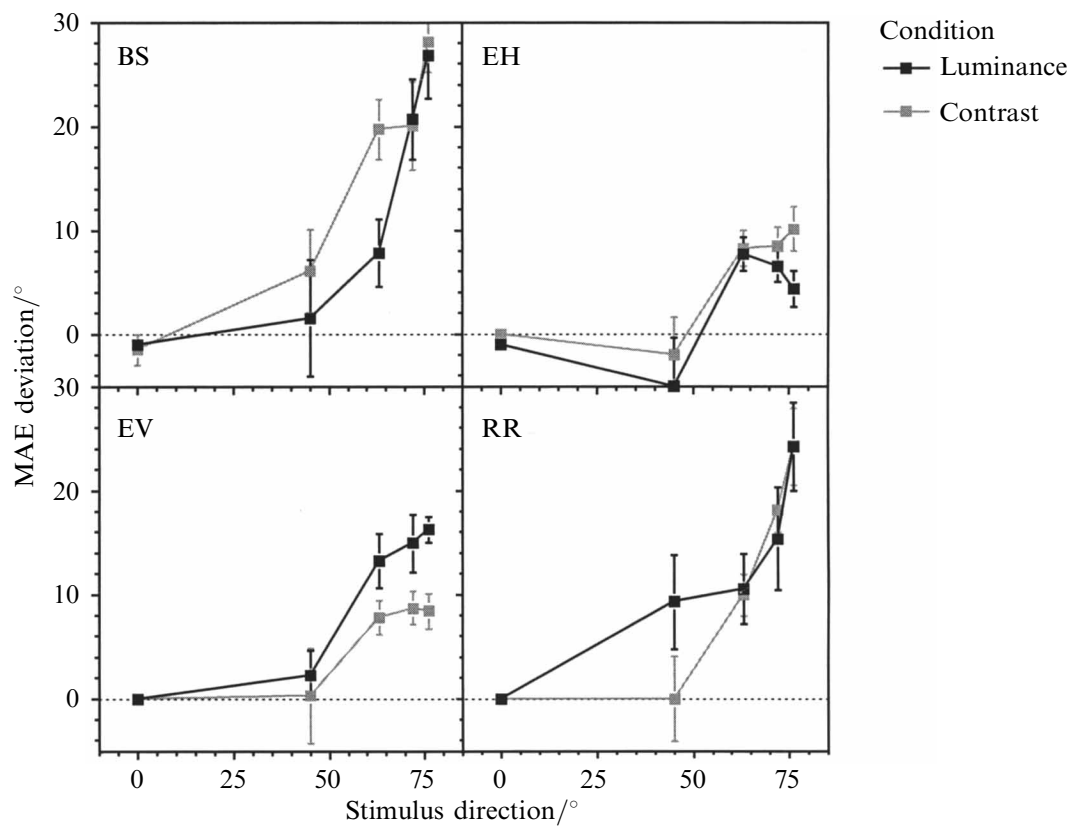

Figure 3. MAE deviations as a function of stimulus direction for four observers for the Luminance (black symbols) and the Contrast (grey symbols) conditions. Error bars depict the SEM. Negative values mean that the MAE direction was perceived to be more oblique than the adapting motion direction. MAE deviations at stimulus directions of $63^{\circ}-76^{\circ}$ were significant $(p<0.01$ in most cases) for all observers. That is, the perceived MAE direction deviated significantly from opposite to the perceived motion direction. This deviation was typically towards more perpendicular to the grating orientation. 
Negative values mean that the MAE is perceived to be more oblique than the motion of the grating. This occurs only occasionally and is never statistically significant. In general, the MAE is always directed more towards opposite to the perpendicular than is the case for the perceived motion. Although the magnitude of this deviation differs across observers and conditions, motion direction and MAE direction are significantly different for all observers in both the Luminance and Contrast conditions $(p<0.05$ in all cases) for motion directions of $63^{\circ}, 72^{\circ}, 76^{\circ}$. Figure 4 shows the mean deviation of the MAE for the three observers for luminance and texture conditions. Again these deviations are significant for the more oblique motion directions.

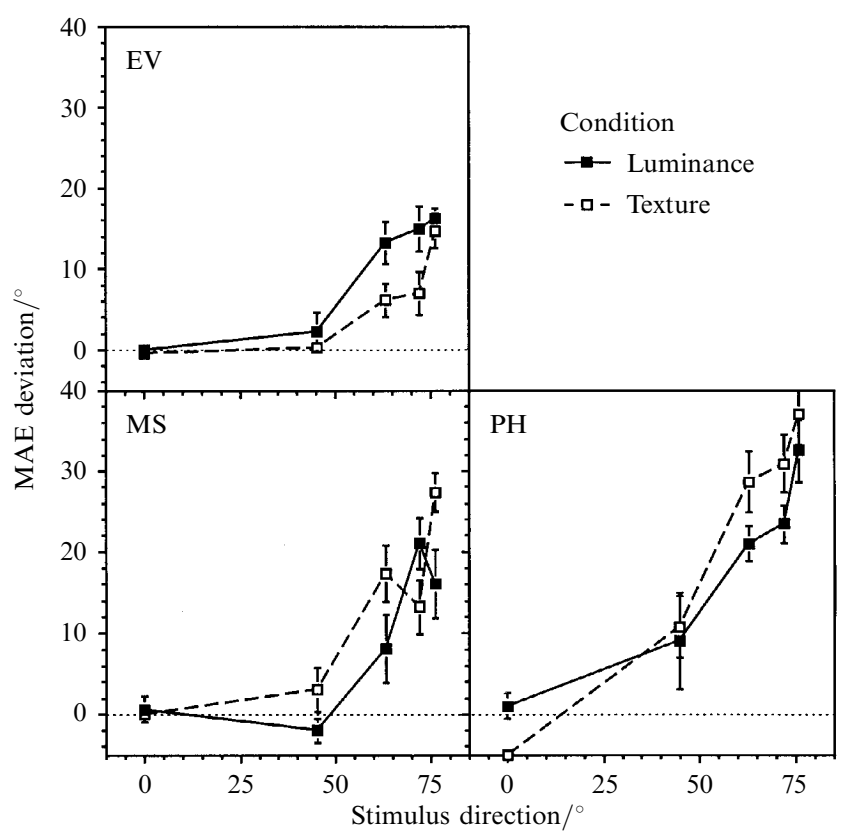

Figure 4. MAE deviations as a function of stimulus direction for three observers for the Luminance (filled symbols) and the Texture (open symbols) conditions. As in figure 3, the MAE deviation was significant ( $p<0.01$ in most cases) for adaptation directions of $63^{\circ}-76^{\circ}$.

\subsection{Discussion}

The results from the Luminance condition of this experiment corroborate the findings by Alais et al (1995). The perceived MAE direction for the more oblique adaptation stimuli differs from the perceived motion direction by as much as $30^{\circ}$ for some observers. Although there is considerable variation between observers, they all show the effect. This condition serves as a comparison for the Contrast and Texture conditions. It is clear from figures 3 and 4 that the perceived MAE directions for the more oblique adaptation stimuli in these latter conditions were directed towards more perpendicular to the grating orientation as well. In other words, the borders of the grating regions, which always move straight down, do influence the direction of the MAE, although they are defined solely by second-order characteristics. This holds for both the contrast border as well as the texture border. Only for observer EV this influence is less strong than the influence of a moving luminance border. None of the other observers shows this difference between conditions.

Figure 5 shows the group data for the three conditions (black line for the Luminance, grey line for the Contrast, and dotted line for the Texture condition). The dashed lines indicate the predicted MAE deviation when the MAE would be directly opposite to the perceived adapting direction (horizontal line) or directly opposite to the motion of the 


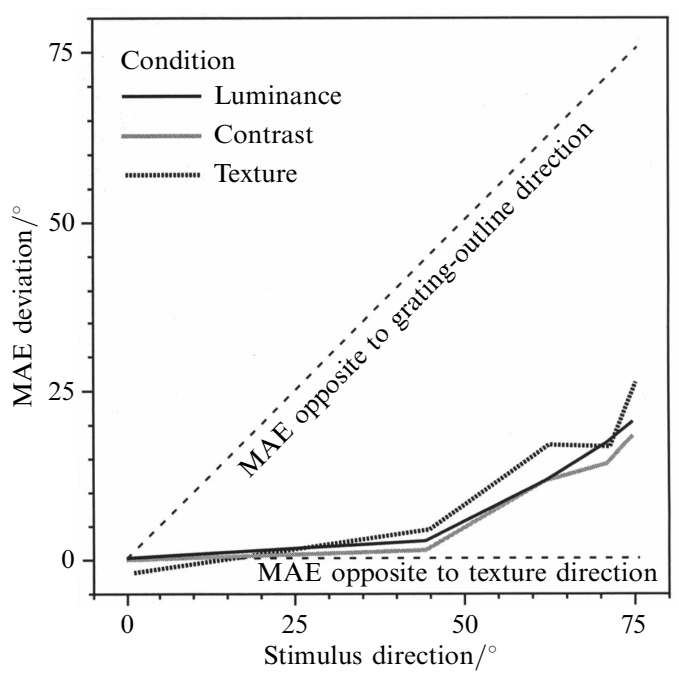

Figure 5. MAE deviations, pooled across observers, for all conditions (Luminance: black line, six observers; Contrast: grey line, four observers; Texture: dotted line, three observers) of experiment 1 . The dashed horizontal line indicates the (absence of) deviation if the MAE would have been determined solely by the oblique motion of the texture. The dashed oblique line indicates the MAE deviation when only the motion of the grating outline (the principal second-order component in the Contrast and Texture conditions) would determine MAE direction.

outline of the grating (oblique line). In the Contrast and Texture conditions, the latter would correspond with a MAE purely opposite to the second-order motion component, while the first would mean a purely first-order MAE. From the figure it is clear that all conditions invoke a similar MAE direction and that the influence of the oblique (texture) motion component decreases with more oblique directions. This might be explained by the fact that the dot displacements increase when the motion direction is further away from perpendicular, while the time between displacements stays equal at $22 \mathrm{~ms}$. Although coherent motion of the texture is still seen at the more oblique directions (hence the fact that the perceived motion direction during adaptation is still close to veridical), this might have affected the strength of the adaptation to the moving texture.

Pilot experiments showed the combined first-order and second-order MAEs for the Texture condition to be very weak and short, so the adaptation and recovery duration were adjusted accordingly (60 s adaptation and $30 \mathrm{~s}$ recovery versus $30 \mathrm{~s}$ adaptation and $60 \mathrm{~s}$ recovery in the other conditions). This ensured confident MAE direction judgments by the observers. A possible explanation for the weaker MAEs for this condition is that this stimulus contained more 'noise', since more mismatches or false correlations can occur for the moving pixels between two consecutive frames (because of the stationary background pixels) during adaptation. Consequently, adaptation to the firstorder motion component in this stimulus might be less effective. Another explanation is based on the fact that all subjects reported seeing motion of the stationary background texture, which was directed opposite to the motion of the moving bars. Since MAEs of this induced motion (eg see Duncker 1929; Reinhardt-Rutland 1988) can occur as well (Anstis and Reinhardt-Rutland 1976; Swanston and Wade 1992; Wade et al 1996) they may have almost nulled the MAEs opposite to the adaptation direction. Longer adaptation duration seemed to solve this problem, although the MAEs were still weaker than those for the Luminance or the Contrast conditions.

\section{Experiment 2}

\subsection{Rationale}

With experiment 1 we have established that the moving texture or contrast border seems to influence the direction of the sMAE, just like the luminance border does. We cannot exclude, however, that the contrast and texture border would have produced an sMAE on their own. In the second experiment, we thus tried to uncouple the first-order and second-order motion components in the stimulus. We created separate first-order and second-order motion gratings, and superimposed them as in a plaid (figure 6). Both 


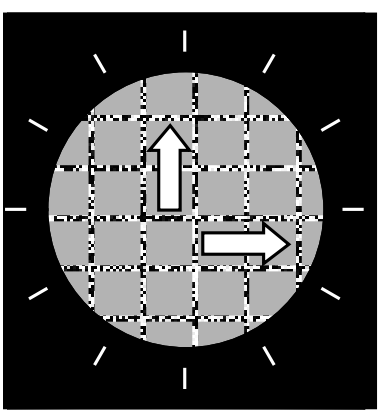

adapt

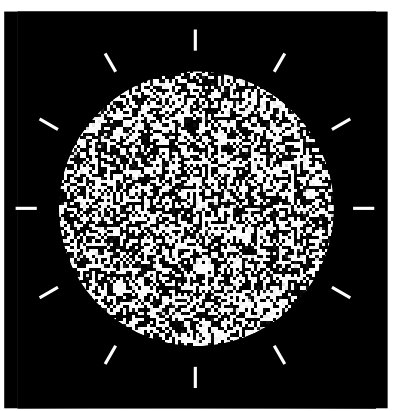

test

Figure 6. Schematic representation of one frame of the adaptation and test stimuli used in experiment 2. Two textured bar patterns were superimposed. The texture of bar pattern could either move coherently with the outline of the bar (first-order motion), which is indicated by the white arrows, or each pixel of the texture could be randomly refreshed on every displacement of the bar pattern (second-order motion). The test pattern was either a static or dynamic field of texture, subtending the entire aperture.

gratings moved perpendicular to their orientation. We tested the MAE direction with stationary texture (as in the Texture condition of experiment 1) and in an additional condition with dynamic texture (which looks like noise on a detuned TV). The influence of the second-order motion component on the MAE direction is expected to be larger for the dynamic than for the static test pattern, since second-order motion alone results in convincing MAEs in dynamic tests, while no or only very weak MAEs have been reported with static test patterns (eg Culham et al 1998). Consequently, when the first-order and second-order motion grating are combined during adaptation, the sMAE (tested with static texture) is expected to be directed more towards the opposite of the first-order motion component than the dMAE (tested with dynamic texture).

\subsection{Methods and procedure}

The apparatus was the same as in experiment 1. Viewing distance was set to $1 \mathrm{~m}$, the circular aperture was $3.7 \mathrm{deg}$ in diameter, and each pixel subtended $1.88 \mathrm{~min}$ of arc. Tick marks were present outside the perimeter of the aperture and the computer keyboard was used to move a pointer on the screen to indicate the MAE direction. Two of the authors and two naïve observers adapted for $45 \mathrm{~s}$ to the two superimposed components while fixating on a dot (15 min of arc in diameter) in the centre of the aperture, after which the test pattern (either static or dynamic) appeared and they had to indicate the MAE direction, or the absence of the MAE when no consistent directional judgment could be made. The pause between trials was $30 \mathrm{~s}$. Motion directions, stimulus combinations, and type of test pattern were presented 8 times to the observers in a semi-random order.

3.1.1 Stimuli. The first-order motion stimulus consisted of 2-pixel-wide textured bars on a grey background which was 30 pixels wide (and had the same mean luminance as the bar texture), so the duty cycle of the grating was $6.25 \%$. The texture moved coherently along with the bars, perpendicular to the bar orientation. The bar was displaced 1 pixel every frame at $90 \mathrm{~Hz}$, resulting in a speed of $2.82 \mathrm{deg} \mathrm{s}^{-1}$. Although there is a second-order motion component in this stimulus (a contrast border), this moves in the same direction and with the same speed as the first-order (texture) motion. The second-order motion stimulus was identical to the first-order stimulus, except that each pixel of the texture was assigned a new random polarity (dark or bright) every frame. There was no net motion energy in the texture of the second-order motion component. Within each single frame, both first-order and second-order components were identical, as can be seen in figure 6 . 
Both first-order and second-order stimuli could be oriented either horizontally or vertically and moved either up or down, or right or left. When the superimposed horizontal and vertical bars were both first-order stimuli, the intersections of two textured regions were refreshed every frame. Under specific conditions (van den Berg and van de Grind 1993; Alais et al 1996), this ensures the perceived coherence of the bars. In the other combinations, the flickering intersections of the bars can be interpreted as part of one or both second-order motion stimuli. The test stimuli were either a stationary texture field with identical spatial properties as the texture on the adaptation stimuli, or this texture field was refreshed every two frames $(45 \mathrm{~Hz})$, thus becoming a dynamic test pattern.

\subsection{Results and discussion}

During adaptation, only the combination of two second-order stimuli yielded a percept of a coherently moving plaid. This is the only condition where the motion of both components is directionally ambiguous. As is the case for a textureless grating viewed through a circular aperture, the only perceived motion direction is perpendicular to the grating orientation, although there is a family of physical grating directions that appear identical. Coherence has been shown to be more likely to occur when the directional uncertainty of the components is large (Lorenceau and Shiffrar 1992; Lorenceau 1996).

The other conditions were perceived as two independent bar patterns sliding over one another, although their MAE was clear and unidirectional. There are conflicting reports about whether first-order and second-order gratings combine into a plaid. Stoner and Albright (1992) constructed a coherent plaid from a first-order and a second-order grating, while Victor and Conte (1992) could not report coherence for a similar stimulus. It appears that these different results depend upon differences in parameters. Since our combination of two first-order motion stimuli already failed to exhibit coherence during adaptation, it is not surprising that our combined first-order and second-order stimuli were perceived sliding as well.

Figure 7 shows the results for the four observers. Depicted are the MAE directions as a function of adaptation stimulus combination (first-order combined with firstorder, first-order combined with second-order, and two second-order stimuli combined) for both a static and a dynamic test pattern. The raw data were converted such that $0^{\circ}$ means that the MAE is exactly opposite to the motion of the vertically oriented component of the stimulus. In the combined first-order and second-order condition, this is always the first-order component. Consequently, $90^{\circ}$ means exactly opposite the horizontal (second-order) component's motion direction.

For the dynamic test pattern, all conditions yield the same results. The dMAE is opposite to a vector sum of both components, irrespective of whether they are first-order or second-order stimuli. Since the MAE direction is around $45^{\circ}$ for all conditions, both second-order and first-order motion components are weighted equally in the dMAE. For the static test pattern, the MAE for the first-order-only condition yielded the same results. For the condition with two second-order motion components, the static test pattern yielded MAEs in about $30 \%$ of the trials. In these cases MAEs were very weak, but directional judgments could be made and were also around $45^{\circ}$. Although an interesting result, for now we treat this condition as one without a MAE.

The most interesting condition is the one where the first-order motion component is combined with a second-order motion component. For the static test pattern, this yields strong MAEs, which are directed opposite to the vector sum of the two components (ie $45^{\circ}$ ). Although the second-order component yields no or only very weak sMAEs when presented in isolation, it is just as strong as the first-order component when combined with the latter in the sMAE. This condition yields the same results as tests with a dynamic test pattern: opposite to a pure vector sum of the motion of both components. 


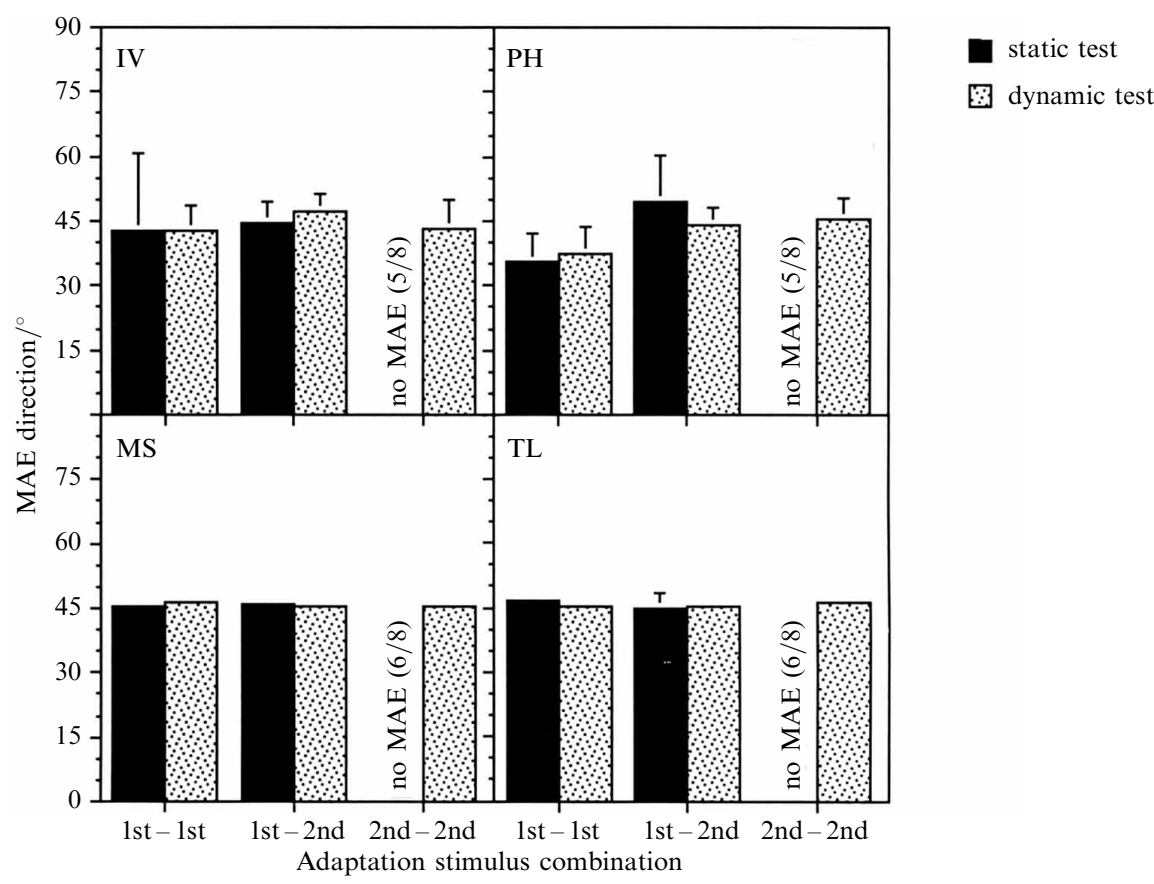

Figure 7. MAE direction plotted for four observers as a function of stimulus combination (firstorder and first-order, first-order and second-order, second-order and second-order) for a static (dark bars) and a dynamic (textured bars) test stimulus. A direction more towards $0^{\circ}$ would mean that the MAE is directed more opposite to the motion of the vertically oriented pattern. In the combined first-order and second-order case, this is the first-order component. All conditions except the second-order-only adaptation and static test condition, yield MAEs of around $45^{\circ}$. For the exception, no clear MAE is perceived in the majority of the trials (5 or 6 out of 8 ).

A similar result can be obtained with a different stimulus configuration, containing a static texture carrier with an added sine-wave grating as first-order component and a multiplied sine-wave grating as second-order component (see Ledgeway 1994). Adaptation to a moving plaid constructed of these gratings (eg Victor and Conte 1992) results in a MAE opposite to the vector sum direction for a stationary plaid test pattern, ie in the same direction as when a dynamic test pattern is used ( $\mathrm{T}$ Ledgeway, personal communication). ${ }^{(3)}$

\section{General discussion}

\subsection{Summary of results}

That second-order motion stimuli can elicit a MAE has been shown before, but typically with dynamic test patterns (eg Culham et al 1998). Threshold elevation studies (eg von Grünau 1986; Turano 1991; Holliday and Anderson 1994), where the detection threshold of a moving stimulus was increased after adaptation to second-order motion in the same direction, but not in the opposite direction, have revealed the adaptation to second-order motion stimuli as well. However, since the threshold of a moving stimulus had to be determined in these studies, they were also dealing with a (special kind of) dynamic test stimulus. The new finding of the present experiments is that, even when a static test pattern is used, the adaptation to second-order motion can still be revealed.

(3) It is interesting to note that a static texture field as test pattern in this case yields little or no sMAE in any direction, which might be due to the different spatial characteristics of the moving adaptation pattern. 
From the experiments it appears that there are certain restrictions. While the dynamic MAE (dMAE) occurs for second-order stimuli just as it occurs for first-order stimuli, a static MAE (sMAE) hardly occurs after adaptation to second-order motion alone. When two second-order gratings are integrated, a hint of an SMAE is there, but it is very weak. These results corroborate previous data [see Culham et al (1998) for an overview] which show that second-order motion elicits only a very weak MAE on stationary test patterns, if any. However, when first-order and second-order motion are both apparent in the adaptation stimulus, their respective adaptations manifest themselves in an integrated, unidirectional sMAE. The manifestation of the adaptation to second-order motion seems just as strong as that for the first-order motion component in the stimulus.

\subsection{Relation to other studies}

Burke and Wenderoth (1993) and Alais et al (1994) adapted to a type-II plaid, of which the gratings were either superimposed and presented simultaneously during adaptation, or they were presented in alternation. They found the sMAE for the superimposed adaptation condition to be opposite to the perceived motion direction, which can be described by a vector summation of all first-order and second-order motions in the stimulus (Wilson et al 1992). The sMAE for the alternating adaptation condition deviated from the perceived plaid direction towards a direction opposite to the one predicted by the intersection-of-constraints rule (Adelson and Movshon 1982). As Burke and Wenderoth as well as Alais et al studied the role of 'blobs' in the perception of moving plaids, they concluded that the absence of the blobs in the alternating adaptation condition accounted for this deviation, and thus that blobs are important in determining plaid direction. Although there is more evidence for a (monocular) blob-tracking mechanism (eg Alais et al 1996, 1997; see also Noest and van den Berg 1993), there might also be another explanation for the differences in sMAE direction after adaptation to superimposed or alternately presented gratings. In the latter case, the motion of second-order boundaries (apparent if the gratings are superimposed) is absent as well. Consequently, in the first-order as well as the second-order domain, the only possible adaptation is to the motion of the grating outline. This would also mean a deviation of the sMAE from directly opposite the perceived plaid direction.

Mather et al (1985) and Nishida and Sato (1995) also used a stimulus that contained both first-order and second-order motion components. However, the first-order and second-order components had opposite directions, so any possible influence on the sMAE of their second-order component was harder to discern. Both Mather and colleagues, and Nishida and Sato reported that the strength of the sMAE (in the direction opposite to the first-order component) decreased as the relative strength of the second-order component in their adaptation stimulus was increased. Thus it seems that adaptation to the second-order motion component did play a role, ${ }^{(4)}$ yet Nishida and Sato showed in their paper that there is no sMAE of second-order motion alone.

\section{2 sMAEs and second-order motion}

Our experiment 2 shows that despite the fact that the second-order motion component is mostly ineffective in eliciting an sMAE on its own, its contribution to the sMAE after combined first-order and second-order adaptation equals that of the first-order motion component. This is a remarkable and counterintuitive result, which is not easy to explain. It is tempting to suggest that some kind of threshold must be crossed before the secondorder motion adaptation becomes apparent in the sMAE. This threshold activity or imbalance might be easier to exceed by a first-order motion stimulus. This would mean, however, that the perceptual manifestation of the sMAE originates from a site where motion information from both first-order and second-order motion is integrated.

(4) Although Mather et al interpreted their data as showing no second-order contribution to the sMAE. 
If the first-order and second-order sMAE would originate from different loci, the firstorder sMAE would not be likely to be modulated by a second-order motion stimulus that by itself does not elicit an sMAE. In a recent experiment, Bertone and von Grünau (1998) reported convincing sMAEs after adaptation to second-order motion in the periphery, although foveal adaptation to second-order motion did not elicit an sMAE. They suggested that foveal second-order motion processing might be a special case. It might be that a possible threshold needed to elicit an sMAE is (much) higher foveally.

\section{3 sMAEs versus dMAEs}

Our present data indicate that a distinction between static and dynamic MAEs does not have to bear on the postulate that the dMAE originates from higher levels of motion processing than the sMAE. Neither do they imply a functional distinction of a separate first-order and second-order motion system. Recent data on adaptation speed (Verstraten et al 1998; van der Smagt et al 1999) demonstrate a distinction in the speed domain, in which both static and dynamic MAEs can stem from low-level (first-order) motion sensors. The latter distinction implies that the sMAE and dMAE originate from (perhaps multiple) parallel loci. Whatever the specific architecture, it is clear from our data that second-order motion is not only available at the site(s) where the dynamic MAE is generated, but at the site(s) responsible for the static MAE as well.

Acknowledgements. MS is supported by the Council of Earth and Life Sciences (ALW) of the Netherlands Organization for Scientific Research (NWO), and FV is a Research Fellow of the Royal Netherlands Academy of Arts and Sciences (KNAW).

\section{References}

Adelson E H, Movshon J A, 1982 "Phenomenal coherence of moving visual patterns" Nature (London) $300523-525$

Alais D, Smagt M J van der, Verstraten F A J, Grind W A van de, 1995 "The perceived direction of textured gratings and their motion aftereffects" Perception $241383-1396$

Alais D, Smagt M J van der, Verstraten F A J, Grind W A van de, 1996 "Monocular mechanisms determine plaid motion coherence" Visual Neuroscience 13 615-626

Alais D, Wenderoth P, Burke D, 1994 "The contribution of one-dimensional motion mechanisms to the perceived direction of drifting plaids and their after effects" Vision Research 34 1823-1834

Alais D, Wenderoth P, Burke D, 1997 "The size and number of plaid blobs mediate the misperception of type-II plaid direction" Vision Research 37 143-150

Anstis S M, Reinhardt-Rutland A H, 1976 "Interactions between motion aftereffects and induced movement" Vision Research $161391-1394$

Anstis S M, Verstraten F A J, Mather G, 1998 "The motion aftereffect" Trends in Cognitive Sciences $2111-117$

Berg A V van den, Grind W A van de, 1993 "Do component motions recombine into a moving plaid percept?" Experimental Brain Research 93312 - 323

Bertone A, Grünau M W von, 1998 "Static and dynamic motion aftereffects of first-and secondorder motion in central and peripheral fields of vision" Investigative Ophthalmology \& Visual Science 39(4) S1082

Bowd C, Rose D, Phinney R E, Patterson R, 1996 "Enduring stereoscopic motion aftereffects induced by prolonged adaptation" Vision Research $363655-3660$

Burke D, Wenderoth P, 1993 "Determinants of two-dimensional motion aftereffects induced by simultaneously- and alternately-presented plaid components" Vision Research 33351 - 359

Cavanagh P, Mather G, 1989 "Motion: The long and short of it" Spatial Vision 4 103-129

Cropper S J, Hammett S T, 1997 "Adaptation to motion of a second-order pattern: the motion aftereffect is not a general result" Vision Research 37 2247-2259

Culham J C, Nishida S, Ledgeway T, Cavanagh P, Grünau M W von, Kwas M, Alais D, Raymond J E, 1998 "Higher-order effects", in The Motion Aftereffect: A Modern Perspective Eds G Mather, F A J Verstraten, S M Anstis (Cambridge, MA: MIT Press) pp 85-124

Derrington A M, Badcock D R, 1985 "Separate detectors for simple and complex grating patterns?" Vision Research $251869-1878$

Duncker K, 1929 "Über induzierte Bewegung” Psychologische Forschung 12 180-259

Grünau M W von, 1986 "A motion aftereffect for long-range stroboscopic apparent motion" Perception \& Psychophysics $\mathbf{4 0} 31-38$ 
Grünau M W von, Dubé S, 1992 "Comparing local and remote motion aftereffects" Spatial Vision $6303-314$

Hiris E, Blake R, 1992 "Another perspective on the visual motion aftereffect" Proceedings of the National Academy of Sciences of the USA 899025 -9028

Holliday I E, Anderson S J, 1994 "Different processes underlie the detection of second-order motion at low and high temporal frequencies" Proceedings of the Royal Society of London, Series B $257165-173$

Ledgeway T, 1994 "Adaptation to second-order motion results in a motion aftereffect for directionally-ambiguous test stimuli" Vision Research 342879 -2889

Ledgeway T, Smith A T, 1994 "The duration of the motion aftereffect following adaptation to first-order and second-order motion" Perception 231211 - 1219

Lehmkuhle S W, Fox R, 1976 "On measuring interocular transfer" Vision Research 16428 - 430

Lorenceau J, 1996 "Motion integration with dot patterns: effects of motion noise and structural information" Vision Research $363415-3427$

Lorenceau J, Shiffrar M, 1992 "The influence of terminators on motion integration across space" Vision Research $32263-273$

McCarthy J E, 1993 "Directional adaptation effects with contrast modulated stimuli" Vision Research $332653-2662$

Mather G, 1980 "The movement aftereffect and a distribution-shift model for coding the direction of visual movement" Perception $9379-392$

Mather G, 1991 "First-order and second-order visual processes in the perception of motion and tilt" Vision Research $31161-167$

Mather G, Cavanagh P, Anstis S M, 1985 "A moving display which opposes short-range and long-range signals" Perception $14163-166$

Mather G, Harris J P, 1998 "Theoretical models of the motion aftereffect", in The Motion Aftereffect: A Modern Perspective Eds G Mather, F A J Verstraten, S M Anstis (Cambridge, MA: MIT Press) pp $157-185$

Moulden B, 1980 "After-effects and the integration of patterns of neural activity within a channel" Philosophical Transactions of the Royal Society of London, Series B 290 39-55

Nishida S, Ashida H, Sato T, 1994 "Complete interocular transfer of motion aftereffect with flickering test" Vision Research $342707-2716$

Nishida S, Sato T, 1995 "Motion aftereffect with flickering test patterns reveals higher stages of motion processing" Vision Research $35477-490$

Noest A J, Berg A V van den, 1993 "The role of early mechanisms in motion transparency and coherence" Spatial Vision $7125-147$

Patterson P, Bowd C, Phinney R E, Pohndorf R, Barton-Howard W J, Angilletta M, 1994 "Properties of the stereoscopic (cyclopean) motion aftereffect" Vision Research $341139-1147$

Raymond J E, 1993 "Complete interocular transfer of motion adaptation effects on motion coherence thresholds" Vision Research 33 1865-1870

Reinhardt-Rutland A H, 1988 "Induced movement in the visual modality: An overview" Psychological Bulletin $10357-71$

Smagt M J van der, Verstraten F A J, Grind W A van de, 1999 "A new transparent motion aftereffect" Nature Neuroscience $2595-596$

Steiner V, Blake R, Rose D, 1994 "Interocular transfer of expansion, rotation, and translation motion aftereffects" Perception $231197-1202$

Stoner G R, Albright T D, 1992 "Motion coherency rules are form-cue invariant" Vision Research $32465-475$

Swanston M T, Wade N J, 1992 "Motion over the retina and the motion aftereffect" Perception $21569-582$

Turano K, 1991 "Evidence for a common motion mechanism of luminance-modulated and contrastmodulated patterns: selective adaptation" Perception 20 455-466

Verstraten F A J, Fredericksen R E, van de Grind W A, 1994 "Movement aftereffect of bi-vectorial transparent motion" Vision Research $34349-358$

Verstraten F A J, Smagt M J van der, Grind W A van de, 1998 "Aftereffect of high speed motion" Perception $271055-1066$

Victor J D, Conte M M, 1992 "Coherence and transparency of moving plaids composed of Fourier and non-Fourier gratings" Perception \& Psychophysics 52 403-414

Wade N J, Spillmann L, Swanston M T, 1996 "Visual motion aftereffects: critical adaptation and test conditions" Vision Research $362167-2175$

Wilson H R, Ferrera V P, Yo C, 1992 "A psychophysically motivated model for two-dimensional motion perception" Visual Neuroscience 9 79-97 


\section{APPENDIX}

Interocular transfer (IOT) of the MAE for static and dynamic test patterns was measured for two observers, who adapted $45 \mathrm{~s}$ to a coherently moving texture pattern, which was presented to the right eye only. A grey screen with the same mean luminance as the adapting pattern was presented to the left eye. This grey screen has been reported to enhance IOT (Lehmkuhle and Fox 1976). The test pattern (either static or dynamic visual noise; see figure 6 , right panel) was presented to the right eye (Mon - Mon condition) in half of the trials. In the other trials the left eye was tested (IOT condition). In the latter case the grey screen was presented to the right eye during the test. Each condition (Mon-Mon versus IOT, and static versus dynamic pattern) was tested eight times; the adapting pattern moved leftward in half of the trials and rightward in the other half. Between trials there was a $30 \mathrm{~s}$ recovery period. Figure A1 clearly shows that the Mon-Mon condition always yields longer MAEs for both the static and the dynamic test pattern, which can be explained by the larger contribution to the MAE of cortical units driven by only one eye (see Moulden 1980). It is clear that static and dynamic test patterns in this experiment yield a similar (partial) IOT.

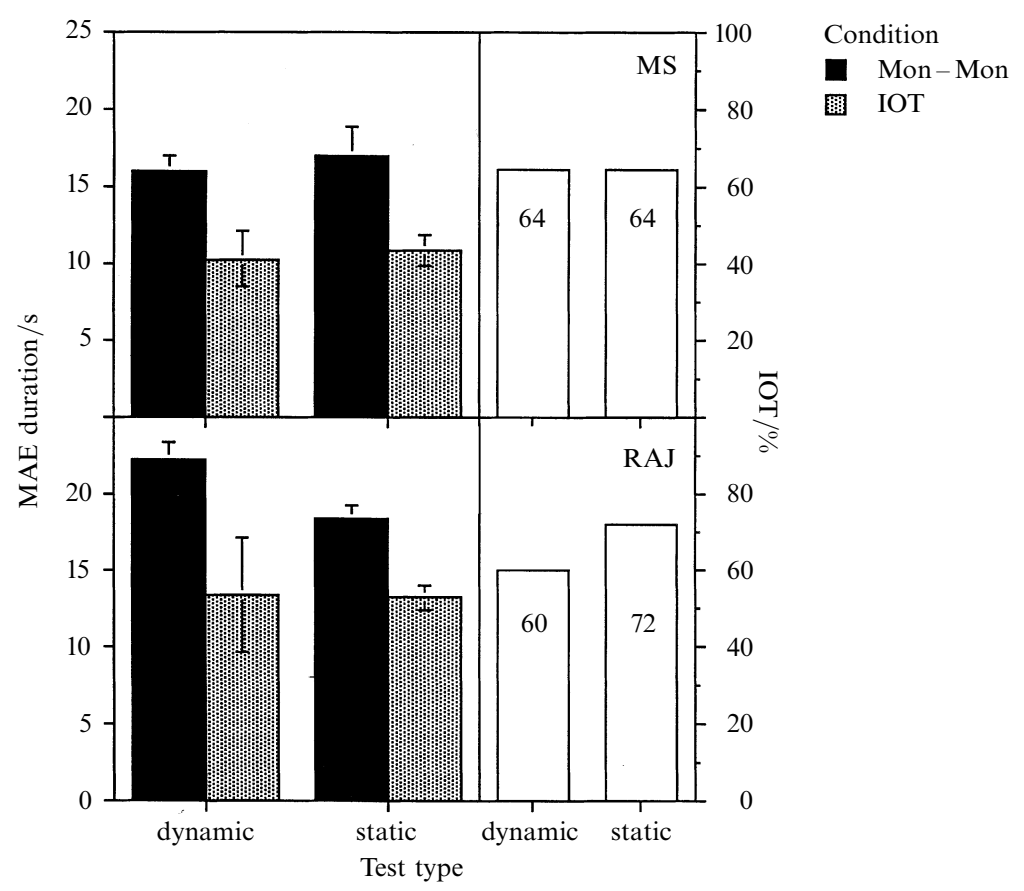

Figure A1. Interocular transfer (IOT) of the MAE for a static and a dynamic test pattern for two observers. Left panels: Dark bars represent the mean MAE duration when the right eye was tested (the Mon-Mon condition), whereas grey bars represent the mean MAE duration when the left eye was tested (IOT condition). Error bars represent the SEM. Right panels: The percentage IOT shows that static and dynamic test patterns yield a similar IOT. 